\title{
Synaptic clustering during development and learning: the why, when, and how
}

\author{
Johan Winnubst and Christian Lohmann* \\ Department of Synapse and Network Development, Netherlands Institute for Neuroscience, Amsterdam, Netherlands
}

\section{Edited by:}

James Jontes, Ohio State

University, USA

Reviewed by:

Hansen Wang, University of

Toronto, Canada

Cristopher Niell, University of

Oregon, USA

*Correspondence:

Christian Lohmann, Department of

Synapse and Network

Development, Netherlands Institute

for Neuroscience, Meibergdreef 47,

Amsterdam, Netherlands.

e-mail:c.lohmann@nin.knaw.nl
To contribute to a functional network a neuron must make specific connections and integrate the synaptic inputs that it receives in a meaningful way. Previous modeling and experimental studies have predicted that this specificity could entail a subcellular organization whereby synapses that carry similar information are clustered together on local stretches of dendrite. Recent imaging studies have now, for the first time, demonstrated synaptic clustering during development and learning in different neuronal circuits. Interestingly, this organization is dependent on synaptic activity and most likely involves local plasticity mechanisms. Here we discuss these new insights and give an overview of the candidate plasticity mechanisms that could be involved.

Keywords: synapse development, synaptic plasticity, dendrites, dendritic integration, activity-dependent, spontaneous activity

\section{INTRODUCTION}

Our brains can adjust to the challenges and opportunities in our environment by activity-dependent adaptations of neuronal connectivity. In particular, during brain development networks undergo activity regulated remodeling at high rates. Interestingly, neuronal activity helps to set up the connections between nerve cells in our brains already before birth and the onset of experience. At these early developmental stages networks generate spontaneous activity that is transmitted along neuronal pathways to test-run and fine-tune the emerging synaptic connections (Goodman and Shatz, 1993; Katz and Shatz, 1996; Ben Ari, 2001; Cline, 2003; Hua and Smith, 2004; Huberman et al., 2008; Sanes and Yamagata, 2009). Thus, activity-dependent fine-tuning prepares brain circuits for the moment when humans and animals start interacting with their environments.

Spontaneous activity occurs frequently as repetitive network events during which large proportions of the network become activated at the same time (Galli and Maffei, 1988; Ben-Ari et al., 1989; Meister et al., 1991; Yuste et al., 1992; O'Donovan et al., 1994; Garaschuk et al., 1998). The activity propagates through the network in a wave-like fashion as neighboring cells become successively activated. This specific characteristic of spontaneous activity has been recognized as an important feature for the activity-dependent establishment of synaptic specificity. For example, waves of spontaneous activity in the vertebrate retina lead to a high degree of correlation between the activity patterns of neighboring cells. Thus, the spatial relationship of the retina is encoded by the activity patterns in higher order brain structures aiding retinotopic map formation and eye specific segregation. Indeed, studies that affected not the presence of spontaneous activity but the patterns within the activity itself have shown an instructive role of spontaneous activity during development
(Weliky and Katz, 1997; Mrsic-Flogel et al., 2005; Torborg et al., 2005; Xu et al., 2011).

Thus, both spontaneous activity before the onset of sensory experience as well as neuronal activity during learning processes (Chklovskii et al., 2004; Hofer et al., 2006; Karmarkar and Dan, 2006; DeBello, 2008; Fu and Zuo, 2011) set up and modify connection specificity on the level of cell-types and individual neurons. A recent series of studies have predicted that this specificity could extend far beyond simply connecting the right axon with the correct cell and might entail a more precise subcellular organization. The prediction stems from both modeling and experimental studies in which individual segments of dendrite are thought to function as independent computational subunits (Polsky et al., 2004; Losonczy and Magee, 2006; Larkum and Nevian, 2008; Branco and Hausser, 2010). In this model, synapses that encode similar information are clustered close together on the dendrite. Recent findings suggest that this clustered organization could be established through local plasticity mechanisms during development and learning. This review aims to give an overview of the research behind these new insights.

\section{THE DENDRITIC COMPARTMENTALIZATION MODEL}

The dendritic tree receives the bulk of synaptic inputs and plays an important role in the integration of incoming signals. It is, therefore, not surprising that the way the dendrite processes synaptic activity to influence somatic firing has been the topic of much research and debate (Poirazi and Mel, 2001; Poirazi et al., 2003; Yuste, 2011). In the classical view the dendrite is seen as a linear integrator, summing the received inputs independently of their position on the dendritic tree as they are transmitted toward the soma. When the linear sum of these inputs reaches a certain threshold value at the soma a non-linear processing step 
takes place as an action potential is generated in an all-or-none fashion (Figure 1). In this model, known as the integrate-andfire model (Abbott, 1999), the impact of a single synapse on somatic firing is low since it is merely one input among many available ones. Consequently, the information a single-cell stores is represented in the changing patterns of synaptic weights spanning the entire cell. It is clear that for this "synaptic democracy" (Yuste, 2011) to work the dendrite must propagate and integrate the synaptic signals in a linear and neutral way. Interestingly, recent studies showed that dendrites contain ionic conductances capable of generating active dendritic events in response to local synaptic activity that can mediate non-linear synaptic integration (Schiller et al., 1997; Hausser et al., 2000; Nevian et al., 2007; Yuste, 2011). For example, voltage-gated calcium channels, sodium channels, and NMDA receptor channels facilitate regenerative events that can spread along the entire dendrite. In particular, dendritic NMDA receptor channels can be activated by the synchronous activation of spatially clustered synapses, due to the voltage-sensitive release of the $\mathrm{Mg}^{2+}$ block along a 10-20 $\mu \mathrm{m}$ stretch of dendrite (Losonczy and Magee, 2006; Nevian et al., 2007). The charge generated by such a regenerative "NMDA spike" has been shown to be much larger than the linear sum of the synapses involved. Furthermore, the extended time course of NMDA activation causes the charge to be more effectively passed along toward the soma. Even though one NMDA spike might not be sufficient to trigger an action potential, the influence of these spatially clustered synapses on somatic firing is hereby significantly increased. Finally, these non-linear integration properties of dendrites can be adjusted by local plasticity mechanisms (Losonczy et al., 2008).
The described findings show that, much like the non-linear process of action potential generation near the soma in the axon initial segment (Kole and Stuart, 2012), the synchronous activation of neighboring synapses on a sub-branch of the dendrite can lead to their non-linear summation (Figure 1). This allows for the implementation of a spatio-temporal coding scheme, resulting in a greater specificity in spiking responses and increased computational capabilities. A new model of synaptic integration has, therefore, been proposed in which the dendritic tree consists of local compartments, each functioning as an individual computational subunit (Hausser and Mel, 2003; Govindarajan et al., 2006; Larkum and Nevian, 2008; for an opposing view see: Yuste, 2011). Modeling studies have shown that neurons utilizing compartmentalized synaptic integration can perform transformations that would normally require multiple neurons connected in a network (Poirazi and Mel, 2001; Wu and Mel, 2009). For instance, models of non-linear integrating neurons that were trained on a pattern recognition problem outperformed their linear integrating counterparts by a factor of 46, correctly learning 27,400 vs. 600 patterns (Poirazi and Mel, 2001). Since these models of synaptic integration are not mutually exclusive it is likely that neurons utilize both linear (Yuste, 2011) and nonlinear synaptic integration schemes for different computational tasks.

\section{DIRECT EVIDENCE FOR SYNAPTIC CLUSTERING}

While the dendritic compartmentalization model increases the computational capabilities of a neuron, it places additional demands on the synaptic organization that is established during development. Namely, it requires synapses with a synchronized

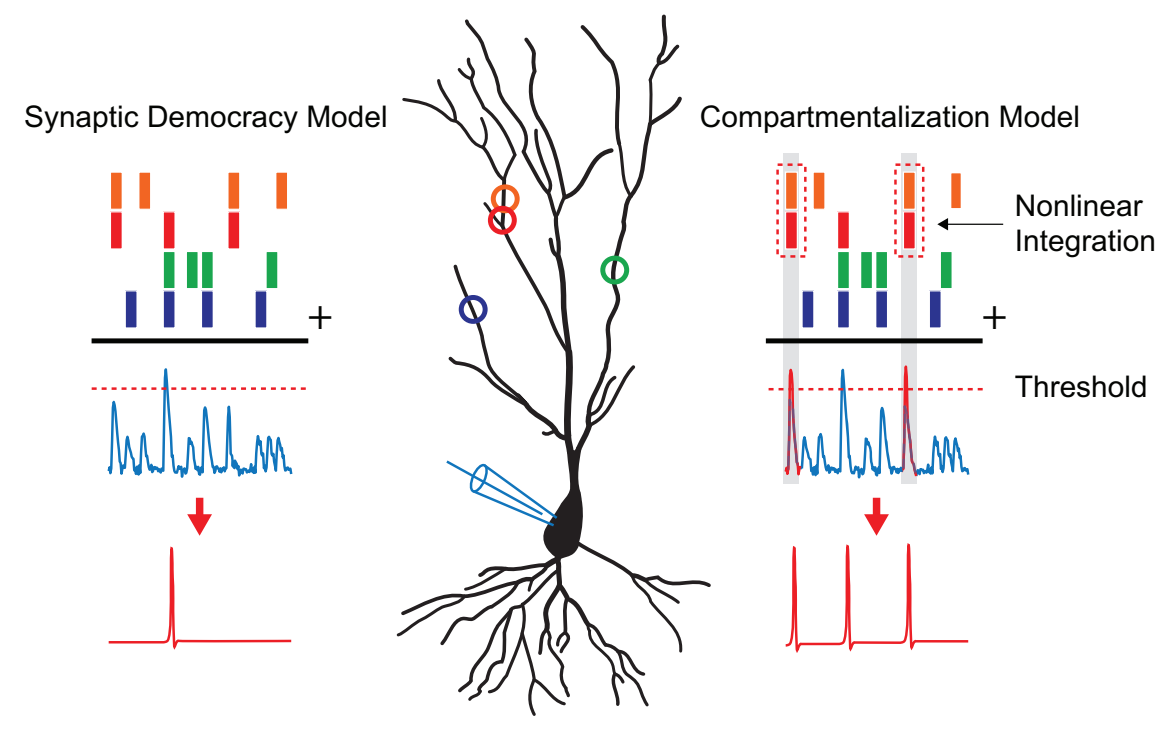

FIGURE 1 | Models of dendritic integration. A pyramidal neuron is depicted with synaptic inputs, represented as colored circles, making distributed contacts along its dendrite. The process of dendritic integration according to the "synaptic democracy" and the "compartmentalization" model are shown. Time points of synaptic input events are represented by the colored bars on top. The blue traces underneath show excitatory postsynaptic potentials as one would record in current clamp. When synaptic inputs reach a certain threshold value (dashed line) an action potential is generated (shown in red) in a non-linear fashion. In the case of the synaptic democracy model, integration is independent of the location of the synaptic inputs. However, when two neighboring synapses are active at the same time in the compartmentalization model (time points highlighted in gray) an additional non-linear step takes place as these inputs are super linearly summated giving them a larger influence on somatic firing. 
activity pattern to be clustered close together on the dendrite. Empirical proof that neurons actually adopt such a synaptic organization has long been lacking. Excitingly, a number of papers have been published recently describing synaptic clustering during development and learning.

\section{PLASTICITY OF SYNAPTIC CLUSTERING IN THE OWL AUDITORY SYSTEM}

The first study on synaptic clustering in vivo was performed in the barn owl auditory localization pathway located in the inferior colliculus (McBride et al., 2008). In this pathway, axons from the lateral shell of the central nucleus (ICCls) send auditory information to the external nucleus (ICX). Here, neurons form a topographic map based on the interaural time difference in the auditory signal received from both ears (Brainard and Knudsen, 1993). Interestingly, a prior study demonstrated that a chronic shift in the visual field, caused by "prism-rearing" of owls, lead to a matching shift in the topographic map of the ICX (DeBello et al., 2001). In this way, visual information functions as an active instructor signal to guide the formation of the auditory spatial map.

The authors speculated that a postsynaptic neuron in the ICX is "taught" to distinguish between input patterns of different spatial locations through the gradual clustering of co-active synaptic inputs. In this case, synaptic contacts that match the spatial tuning of the postsynaptic cell are more likely to be clustered close together and thereby increase their influence on somatic firing. To investigate this possibility an anterograde tracer was used to label ICCls neurons known to represent between 0 and $20^{\circ}$ of auditory space. Since the axonal tree of single ICCls neuron spans a large region of the topographic map in the ICX, its co-active synaptic contacts can either match or mismatch the local spatial tuning depending on their relative location. In the case of prism-reared owls this means that every set of synapses from an ICCls neuron falls along an anatomical continuum of adaptation to the shifted tuning of the postsynaptic cells. Their axonal arbors were, therefore, separated into three zones: a central "normal zone" that was located in the matching $0-20^{\circ}$ ICX region of the normal topographic map, a lateral "adaptive zone" located in the $0-20^{\circ}$ region of the shifted topographic map and a opposing lateral "maladaptive" zone that did not match the tuning of the ICX region in both conditions. Interestingly, the results showed that axo-dendritic contacts in the adaptive zone of prism-reared owls were significantly closer together than those in the normal or maladaptive zone (McBride et al., 2008). These findings could not be explained by a general increase in the number of synapses, because their number remained constant between the different zones. Closer examination showed that inter-contact distances in the adaptive zone were all smaller than $20 \mu \mathrm{m}$. This corresponded with the distances between axonal contacts observed in the central adaptive zone of normal juvenile owls. The shift in the spatial field observed in prism-reared owls, therefore, matched the shift in the localization of clustered synaptic inputs. These findings were the first to suggest that experience can drive the clustering of co-active synaptic inputs in order to modulate sensory responses in a behaviorally relevant way (McBride et al., 2008).

\section{SYNAPTIC CLUSTERING DRIVEN BY SPONTANEOUS ACTIVITY}

As described above, developing neuronal systems generate spontaneous "bursts" of activity where large parts of the network become active in synchrony (Feller, 1999; Wong, 1999; Ben Ari, 2001). To investigate whether clustering is present at this early stage of development, a recent study by Kleindienst et al. (2011) mapped the synaptic input patterns that are received by a single CA3 pyramidal neuron during spontaneous activity in vitro. High speed calcium imaging was combined with whole-cell electrophysiology in order to indentify local calcium transients on the apical dendrite that corresponded with synaptic activation. Synaptic calcium transients occurred at glutamatergic synapses as their activity was sensitive to application of the NMDA receptor antagonist APV.

While the synapses that became activated during a spontaneous network event were found to vary from burst to burst, analysis of the spatio-temporal activity patterns revealed a clear underlying principle: synapses that were closer together on the dendrite $(<16 \mu \mathrm{m})$ were more often co-active then synapses that were farther apart. Anatomical reconstruction of presynaptic axons showed that this observation could not be explained by individual axons making multiple neighboring contacts since axons were found to make a maximum of one contact per postsynaptic cell at this point in development. Furthermore, minimal stimulation of presynaptic axons, that activated single synapses, never lead to the co-activation of neighboring synapses thereby excluding the possibility of spill-over or internal diffusion of signaling factors. These findings suggest that already in this early stage of development (first postnatal week) synapses are organized into local clusters on the dendrite that receive related information (Kleindienst et al., 2011).

Since spontaneous network activity is thought to play a role in establishing connection specificity, the authors speculated that this subcellular organization could be caused by an activitydependant sorting process during development. To investigate this possibility, a separate set of experiments was performed where TTX was applied to the culture medium during incubation. As predicted, neurons that developed in the absence of spiking activity showed no sign of synaptic clustering after normal network activity was restored. Similarly, synaptic clustering was completely abolished in cells that developed in the presence of the NMDAR antagonist APV, suggesting a role for NMDA-dependent plasticity mechanisms. Thus, spontaneous network activity during development is able to connect neurons with subcellular precision. Specifically, synapses that carry similar information are preferentially clustered together on the dendrite in a process that involves NMDAR-dependent plasticity mechanisms (Kleindienst et al., 2011).

Takahashi et al. (2012) described similar results in older rat hippocampal slice cultures (third postnatal week compared to first week). At this age most synapses are located on spines in contrast to the higher number of spineless synapses observed in the first postnatal week (Kleindienst et al., 2011). Synaptic activity could, therefore, be observed as spontaneous calcium transients confined to single spines after somatic patching with a calcium dye. Similarly, these calcium transients were correlated with EPSPs and sensitive to APV application. Furthermore, when 
the activity patterns of these spines were analyzed an almost identical spatio-temporal clustering could be observed. Specifically, spines that were within $8 \mu \mathrm{m}$ of each other were more often coactive within $100 \mathrm{~ms}$. Again, this clustering effect was found to be dependent on NMDA activity as incubation with APV completely abolished synaptic clustering. Similar recordings made by the authors in fast-spiking parvalbumin neurons showed no signs of clustering, indicating that synaptic clustering is a celltype specific characteristic. Taken together, these findings suggest that clusters of co-active synapses are not transiently present during a brief period in development but are maintained into more mature stages of the network (Takahashi et al., 2012). This was further confirmed in an additional in vivo experiment performed in layer $2 / 3$ of the barrel cortex of anaesthetized young adult mice. Yet again, spontaneous co-activity could often be detected in the spines of these dendrites and this occurrence increased when spines were within a $6 \mu \mathrm{m}$ stretch of dendrite. Synaptic clustering is thereby shown to be present in live animals.

While the studies described above revealed that spontaneously co-active synapses are clustered on dendrites it remains unclear to what extent functionally clustered inputs are relevant for sensory processing. In vivo studies performed in the visual, auditory, and somatosensory cortex of adult mice (Jia et al., 2010; Chen et al., 2011; Varga et al., 2011) showed that short stretches of dendrite contain synapses tuned for different aspects of each modality (e.g., orientation preference or tone frequency). While in some instances inputs may be clustered, a general conclusion cannot be drawn, yet. A fascinating question for future in vivo studies is which stimulus characteristics may be encoded in synaptic clusters along dendrites of cortical neurons for different modalities. The answer to this question will have important consequence for our understanding of how sensory inputs are processed in cortical networks.

Comparing the spatial extent of synaptic clustering across the different experimental conditions and developmental ages seems to hint toward a relationship between the increase of synaptic density with age and increased spatially confined synaptic clusters (e.g., 16 and $8 \mu \mathrm{m}$ at one and three postnatal weeks, respectively, Kleindienst et al., 2011; Takahashi et al., 2012). This may be due to increased competition between neighboring synapses resulting in sharper defined synaptic clusters (see also below; Govindarajan et al., 2006).

\section{EXPERIENCE AND LEARNING-DRIVEN SYNAPTIC CLUSTERING IN THE CORTEX}

Since the clustering of synaptic inputs is activity-dependent and most likely involves NMDA-dependent long-term potentiation (LTP) could this mean that synaptic plasticity is similarly clustered on the dendrite? Makino and Malinow (2011) aimed to identify the existence of such clustered plasticity in vivo. They investigated the incorporation of fluorescently labeled AMPA receptor subunits GluR1 and GluR2 in the postsynaptic membrane. DNA constructs for these proteins were delivered into layer $2 / 3$ pyramidal neurons in the mouse barrel cortex through in utero electroporation. Targeted cells expressed either the GluR1 or Glur2 subunit coupled to a $\mathrm{pH}$-sensitive form of GFP (Super Ecliptic pHluorin, SEP-GluR1 or SEP-GluR2, respectively) ensuring exclusive fluorescence of the surface expressed subpopulation. Synaptic incorporation of GluR1 and GluR2 were studied separately since the two subunits are involved in different forms of plasticity. Increased exocytosis of GluR1 to the synaptic membrane is known to be specifically involved in the expression of LTP (Takahashi et al., 2003; Feldman and Brecht, 2005). Conversely, GluR2 is not involved in LTP but is important for homeostatic scaling after deprived activity (Gainey et al., 2009). In accordance with these known differences SEP-GluR2 expression was found to be more enriched in spines of mice that had undergone sensory deprivation by whisker-trimming (Makino and Malinow, 2011). Also, enrichment of SEP-GluR1 was higher in spines of mice that had intact whiskers, confirming a role of GluR1 in the expression of activity-dependent LTP. The authors reconstructed dendrites of individual neurons and showed that enrichment of SEP-GluR1 in nearby spines was correlated. Neighboring spines tended to express similar levels of synaptic plasticity, demonstrating that clustered plasticity also occurs in vivo. Furthermore, this effect was significantly greater in animals with intact whiskers, suggesting that clustering is dependent on normal synaptic activity. Conversely, no signs of SEP-GluR2 clustering were observed in whiskertrimmed animals even though overall levels were significantly higher. Homeostatic scaling after sensory deprivation, therefore, seems to be achieved by a separate cell-wide increase of synaptic potentiation.

The authors argued that the observed clustering of synaptic plasticity can best be explained by a model in which the induction of potentiation at one synapse can activate kinase signaling pathways to lower the threshold for AMPA receptor incorporation at neighboring synapses (see also below; Harvey and Svoboda, 2007; Harvey et al., 2008). To test this hypothesis a similar set of experiments was performed using a mutated form of GluR1 that is insensitive to phosphorylation by PKC/PKA. Fascinatingly, while the global levels of spine enrichment were similar, these mutated forms of SEP-GluR1 showed no signs of dendritic clustering. Clustering of synaptic plasticity, therefore, occurs in freely behaving animals and is directly dependent on intracellular signal transduction in individual stretches of dendrite (Makino and Malinow, 2011). Takahashi et al. (2012) reported a similar finding: they investigated GFP-GluR1 expression in spines of CA1 pyramidal neurons $24 \mathrm{~h}$ after mice were exposed to a novel environment. They found that the probability of observing GFPGluR1-enriched spines was significantly higher when a neighboring spine within $8 \mu \mathrm{m}$ was also GFP-positive. Clustered synaptic plasticity could, therefore, also be observed in the hippocampus in response to natural stimuli (e.g., a novel environment).

Additional evidence for synaptic clustering has recently been found in mice during motor learning. Fu et al. (2012) imaged spinogenesis on layer 5 pyramidal neurons in the motor cortex of Thy-1-YFP-H mice as they were trained in a forelimb motor task. Roughly one-third of the newly formed spines that emerged during the acquisition phase of the training appeared next to another novel spine without interspersed existing spines. Clustering of newly formed spines was significant compared to spine formation in both the untrained control mice and during consolidation. Furthermore, clustered spines $(<5 \mu \mathrm{m})$ had 
a significantly higher survival rate at training day 16 as well as four months after training had stopped. Repetitive motor learning is, therefore, found to induce the long-term formation of clustered synaptic contacts (Fu et al., 2012). Importantly, this clustering was found to be task-specific as only very few spines clustered with spines induced by another motor task. While new emerging spines avoided existing spines, most likely due to competition for local resources, they did emerge very close to previously established new spines-related to the same learning task. Learning-induced synaptic clustering, therefore, may be a consequence of local cooperativity between emerging spines-related to the same task.

\section{PLASTICITY MECHANISMS FAVORING SYNAPTIC CLUSTERING}

The findings discussed so far show that synapses with synchronized activity patterns are preferentially clustered close together on the dendrite. Furthermore, clustering of synaptic inputs is found to be an activity-dependent process during development and learning. Although the exact underlying mechanism remains unclear, the observation that neighboring synapses undergo similar forms of plasticity seem to suggest that local plasticity plays a role. Interestingly, several local plasticity mechanisms have recently been described which could aid the formation of a clustered synaptic organization through activity-dependent processes.

\section{SYNAPTIC TAGGING AND CAPTURE}

Plasticity mechanisms for the establishment of functionally clustered synaptic inputs should act on a local scale within the dendritic arborization. A recently proposed idea is that local plasticity can be mediated by a "synaptic tagging and capture" (STC) mechanism. This mechanism was first described on the level of individual cells in a classic study by Frey and Morris (1997) where they found that cooperation can take place between synapses. Specifically, they found that tetanus field stimulation in the Schaffer collateral-CA1 pathway that induced LTP in one set of synapses facilitated the expression of LTP in a different set of synapses which received a subthreshold stimulus. This effect could even be observed when the two stimulations were separated by more than $1 \mathrm{~h}$. The authors proposed that the induction of LTP leads to the creation of a protein synthesis independent synaptic "tag" which "captures" plasticity-related proteins needed for the induction of LTP (Frey and Morris, 1997). Since plasticity-related proteins are not confined to individual synapses, the proteins sequestered by a synaptic tag do not have to be generated by the same event that caused the setting of the tag.

To fully understand how cooperation between synapses influences the storage of memory engrams it is important to consider where activity-induced protein synthesis takes place. It has been suggested that protein synthesis can occur in ribosomes localized to individual synapses (Steward and Levy, 1982; Steward and Schuman, 2003). Assuming that plasticity-related proteins spread from the location of the synapse whose activity lead to their generation it could be hypothesized that STC preferentially occurs at clusters of neighboring synapses on the dendrite (Govindarajan et al., 2006). A study by Govindarajan et al. (2011) investigated this possibility by identifying the spatio-temporal characteristics of STC at the level of individual spines. To induce LTP at a single spine they combined high-frequency glutamate uncaging with bath application of forskolin (Govindarajan et al., 2011). Conversely, glutamate uncaging in the absence of forskolin resulted in shorter lived potentiation. The authors found, in accordance with the STC model, that stimulation of one spine in the presence of forskolin followed by stimulation of a second spine in the absence of forskolin lead to LTP expression in both spines (Govindarajan et al., 2011). Importantly, the efficiency of STC expression depended on the distance between the two stimulated spines and was almost completely undetectable at $70 \mu \mathrm{m}$ (Figure 2A).

These findings show that STC preferentially occurs at neighboring synapses, making the dendritic branch the preferred site for cooperation and association between synapses. Conversely, since the presence of plasticity-related proteins appear as the ratelimiting factor in LTP, coupled with the observed faster degradation of plasticity-related proteins compared to synaptic tags; it is imaginable that competition takes place between tagged synapses for sparsely available plasticity-related proteins. Indeed, the stimulation of just two spines during STC already resulted in both spines reaching their maximum size more slowly (Govindarajan et al., 2011). In some cases the growth of one stimulated spine could even be correlated with the shrinking of another. The presence of a limited plasticity-related protein pool may, therefore, cause competition between multiple tagged synapses on the same dendritic branch. This kind of competition could explain the previously observed relationship between the increased synaptic density with age and sharper spatially defined synaptic clusters (see above).

\section{METAPLASTICITY THROUGH LOCAL SYNAPTIC ACTIVITY}

The induction of long-term plasticity requires the activation of various signaling cascades in order to stimulate the necessary protein synthesis. One of these signaling cascades involves mitogen-activated protein kinase (MAPK) and mechanistic target of rapamycin (mTOR; Kelleher III et al., 2004a,b). Since MAPK and mTOR remain active for several minutes after activation by plasticity-inducing stimulation, it has been suggested that this pathway could lower the threshold for plasticity in neighboring synaptic activity for a certain period of time (Wu et al., 2001; Govindarajan et al., 2006). Such processes that reflect a higher order level of plasticity, or the "plasticity" of plasticity mechanisms, have collectively been referred to as "metaplasticity" (Abraham et al., 2001; Abraham, 2008). If the threshold for plasticity induction is indeed regulated by local synaptic activity, synapses that are active shortly after LTP induction at a neighboring synapse would have a higher chance of also becoming potentiated. As a consequence, neighboring synapses with similar activity patterns would be preferentially potentiated and stabilized. To test this possibility Harvey and Svoboda (2007) investigated spiketiming-dependent potentiation (STDP) in neighboring spines of hippocampal pyramidal neurons. Here, STDP was induced in single spines using two-photon glutamate uncaging followed by the initiation of three action potentials. Similar to classical experiments of STDP, the magnitude of plasticity was found to decrease 
A Synaptic Tagging and Capture
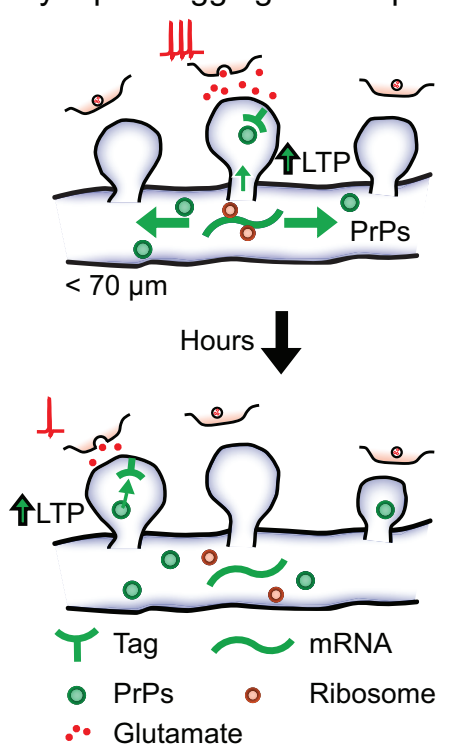

B
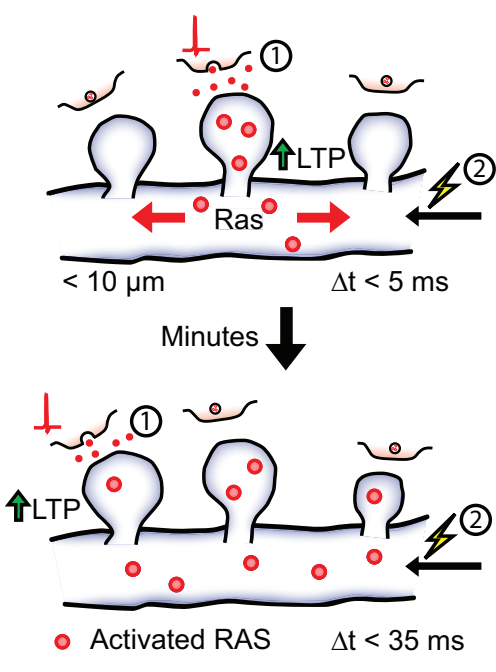

c Activation of Silent Synapses
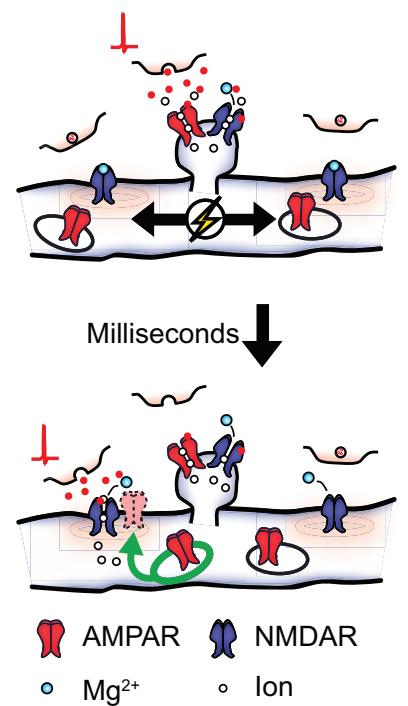

FIGURE 2 | Possible local plasticity mechanisms that establish synaptic clustering. Shown is a local stretch of dendrite containing three synaptic spines. (A) Synaptic tagging and capture. Strong postsynaptic activation leads to the synthesis of plasticity-related proteins (PrPs) that become available in local stretches of dendrite. Synapses tagged within a timescale of hours by weak activation (shown below) can capture plasticity-related proteins in order to express long lasting LTP. (B) Crosstalk. Postsynaptic activation (1) followed by action potential firing (2, spike-timing window $<5 \mathrm{~ms}$ ) leads to the induction of LTP and causes activated RAS from the stimulated spine to spread into the dendrite. The presence of activated RAS increases the spike-timing window ( $<35 \mathrm{~ms}$ ) of later active synapses (timescale of minutes) thereby lowering the threshold for LTP induction. (C) Activation of silent synapses. Shown are two silent synapses present on the dendritic shaft that do not express AMPA receptors on their surface, next to an active synaptic spine. Activation of the non-silent spine leads to a local depolarization of the membrane causing the removal of the $\mathrm{Mg}^{2+}$ block from NMDA receptors in the neighboring synapses. A silent synapse whose presynaptic cell fires coincidently undergoes Hebbian plasticity resulting in the insertion of intracellular AMPA receptors and its eventual stabilization. as the time between glutamate uncaging and action potential initiation increased. Specifically, the increase in uEPSC amplitude and spine volume could not be observed with a spike time window larger than $5 \mathrm{~ms}$. However, it was found that previous induction of LTP in one spine broadened the spike time window of STDP in neighboring spines (up to $35 \mathrm{~ms}$; Figure 2B). No change in STDP was observed when the time between stimulation of the two spines increased up to $10 \mathrm{~min}$ or when their relative distance exceeded $10 \mu \mathrm{m}$. Taken together, these findings show that the threshold for plasticity-induction at an individual spine can be influenced by the activity of neighboring synapses. Unlike STC, the effect was independent of protein synthesis and did not occur when the weak stimulation protocol preceded the LTP protocol (Harvey and Svoboda, 2007). This phenomenon is, therefore, referred to as "crosstalk" in order to distinguish it from STC mechanisms.

One of the early activators of the MAPK signaling pathway is the guanosine triphosphatase (GTPase) Ras. This GTPase becomes activated by NMDAR-induced calcium influx and is involved in the induction of LTP (Zhu et al., 2002). In order to uncover if activated RAS remains confined to single spines a later study by Harvey et al. (2008) used a fluorescence resonance energy transfer (FRET) based indicator of RAS activation. The authors observed a robust increase of RAS activity after LTP induction in stimulated spines that peaked within $1 \mathrm{~min}$ and returned to baseline after $15 \mathrm{~min}$. Interestingly, the magnitude of RAS activation correlated with the observed increase in spine volume while no RAS activation could be observed during a subthreshold LTP protocol. Once activated, RAS spread over several micrometers in both directions of the dendrite and subsequently invaded neighboring spines. Since both the spatial and temporal activation profile of RAS closely matched those observed in the crosstalk experiments the authors speculated that RAS activation could be involved in the expression of crosstalk. This was confirmed in a subsequent experiment where crosstalk was prevented by the local application of a pharmacological blocker for MAPK and extracellular signal-regulated kinase (ERK; Harvey et al., 2008). These findings show that $\mathrm{Ca}^{2+}$-activated signaling machinery can regulate the threshold for plasticity induction in local stretches of dendrite. In this way the occurrence of strong LTP-inducing activity at one synapse can lower the threshold for potentiation at neighboring synapses, thereby aiding the storage of memory engrams in clusters of synapses on the dendrite (Figure 2B). Interestingly, local diffusion $(<5 \mu \mathrm{m})$ has also been observed for the GTPase RhoA upon spine activation while yet another GTPase (CDC42) showed no such spatial characteristics (Murakoshi et al., 2011). Individual elements of the MAPK pathway could, therefore, be differentially involved in the expression of this local plasticity mechanism. 


\section{SILENT SYNAPSES AND AMPA RECEPTOR REGULATION}

Developmental networks are frequently characterized by a high incidence of synapses that can be identified structurally, but - under regular signaling conditions_-do not mediate synaptic transmission. For example, developing synapses can switch between an AMPA signaling state and an AMPA "silent" state (Hanse et al., 2009). In the "silent" state no AMPARs are present on the postsynaptic membrane and the synapse is, therefore, unable to generate an EPSP in native conditions due to the $\mathrm{Mg}^{2+}$ block present in NMDARs (Liao et al., 1995). Since activation of silent synapses requires the depolarization of the local membrane: could the preferential un-silencing of co-active synapses be another possible mechanism for the establishment of synaptic clusters? Once a synapse becomes silent it can be reactivated (unsilenced) by participating in Hebbian-like activity patterns and thereby regaining its AMPA signaling capabilities. It will be interesting to investigate, whether membrane depolarization caused by local synaptic activity is sufficient to lead to NMDA activation in neighboring silent synapses (but not in more distant ones). In this case a silent synapse whose activity is synchronized with the activity of its neighbors will have a higher chance of becoming un-silenced and subsequently stabilized (Figure 2C). Over time this would lead to the establishment of synaptic clusters with similar activity patterns through activity-dependent self-organization.

\section{SUMMARY AND CONCLUSION}

The local plasticity mechanisms discussed here converge on one general theme: the activity of one synapse can modulate the plasticity at neighboring synapses and thereby influence their fate. This can be through the mutual sharing of plasticity-related proteins (STC), adaptation of a local plasticity threshold (metaplasticity), or by the activation of synchronized synaptic inputs (e.g., un-silencing). These mechanisms operate within different temporal domains, ranging from hour to millisecond time scales (Figure 2). Such timing differences may assist the coupling of inputs during different developmental and plasticity paradigms. For example, clustering of coincident synaptic activation during bursts of spontaneous activity mediated by local activation of silent synapses may facilitate connecting inputs with similar signaling properties to a common dendrite during development. On the other hand, learning based on long-term experience may

\section{REFERENCES}

Abbott, L. F. (1999). Lapicque's introduction of the integrate-and-fire model neuron (1907). Brain Res. Bull. 50, 303-304.

Abraham, W. C. (2008). Metaplasticity: tuning synapses and networks for plasticity. Nat. Rev. Neurosci. 9, 387.

Abraham, W. C., Mason-Parker, S. E., Bear, M. F., Webb, S., and Tate, W. P. (2001). Heterosynaptic metaplasticity in the hippocampus in vivo: a BCM-like modifiable threshold for LTP. Proc. Natl. Acad. Sci. U.S.A. 98, 10924-10929.

Belmonte, M. K., Allen, G., BeckelMitchener, A., Boulanger, L. M.,
Carper, R. A., and Webb, S. J. (2004). Autism and abnormal development of brain connectivity. J. Neurosci. 24, 9228-9231.

Ben Ari, Y. (2001). Developing networks play a similar melody. Trends Neurosci. 24, 353-360.

Ben-Ari, Y., Cherubini, E., Corradetti, R., Galarsa, J.-L. (1989). Giant synaptic potentials in immature rat $\mathrm{CA} 3$ hippocampal neurones. J. Physiol. 416, 303-325.

Brainard, M. S., and Knudsen, E. I. (1993). Experience-dependent plasticity in the inferior colliculus: a site for visual calibration of the neural representation of auditory space

require plasticity mechanisms that integrate information over longer periods of time, such as STC.

Together these findings suggest a series of events leading to the establishment and refinement of connectivity with subcellular precision. First, clustering of synchronized synaptic inputs arises through an activity-dependent sorting process during development. Initially, synaptic contacts are established in a (more or less) random fashion along a dendrite. Then, bursts of spontaneous synaptic inputs help to select a portion of these synaptic contacts through local cooperation and competition. Synapses that are temporally synchronized with their neighbors get preferentially strengthened and stabilized through local plasticity mechanisms while synapses that are desynchronized with their surroundings get eliminated. These simple self-organizational principles will over time lead to the establishment of functional clusters of synaptic inputs on the dendrite. During development the established synaptic clusters could function as a principal site for rudimentary pattern recognition, even before sensory information is received. Subsequently, when sensory systems become functional during interactions with the environment, synaptic reorganization continues to adapt synaptic clusters through experience-driven and learning-induced activity.

As described above, clustering functional inputs is most likely required to harvest the full computational power of individual neurons. Therefore, errors in wiring at this level will compromise the capacity of neurons to process information. Interestingly, various cognitive developmental disorders have been linked to alterations in synaptic organization. For instance, neuroanatomical evidence suggests that brains of autism patients show higher levels of local connectivity that impede the formation of long-range connections necessary for central cognitive control (Belmonte et al., 2004; Geschwind and Levitt, 2007). Whether these alterations are due to increased synapse formation or deficits in synapse elimination and pruning are currently unknown. In this light, one might speculate that perturbed synaptic clustering may cause neurocognitive disorders that are associated with deficits in synaptic connectivity (Zoghbi, 2003; Belmonte et al., 2004; Geschwind and Levitt, 2007).

\section{ACKNOWLEDGMENTS}

We thank Christian Levelt, Helmut Kessels, and Maarten Kole for their suggestions and valuable comments on the manuscript.

in the barn owl. J. Neurosci. 13, 4589-4608.

Branco, T., and Hausser, M. (2010). The single dendritic branch as a fundamental functional unit in the nervous system. Curr. Opin. Neurobiol. 20, 494-502.

Chen, X., Leischner, U., Rochefort, N. L., Nelken, I., and Konnerth, A. (2011). Functional mapping of single spines in cortical neurons in vivo. Nature 475, 501-505.

Chklovskii, D. B., Mel, B. W., and Svoboda, K. (2004). Cortical rewiring and information storage. Nature 431, 782-788.
Cline, H. (2003). Sperry and Hebb: oil and vinegar? Trends Neurosci. 26, 655-661.

DeBello, W. M. (2008). Micro-rewiring as a substrate for learning. Trends Neurosci. 31, 577-584.

DeBello, W. M., Feldman, D. E., and Knudsen, E. I. (2001). Adaptive axonal remodeling in the midbrain auditory space map. J. Neurosci. 21, 3161-3174.

Feldman, D. E., and Brecht, M. (2005). Map plasticity in somatosensory cortex. Science 310, 810-815.

Feller, M. B. (1999). Spontaneous correlated activity in developing neural circuits. Neuron 22, 653-656. 
Frey, U., and Morris, R. G. M. (1997). Synaptic tagging and longterm potentiation. Nature 385, 533-536.

Fu, M., Yu, X., Lu, J., and Zuo, Y. (2012). Repetitive motor learning induces coordinated formation of clustered dendritic spines in vivo. Nature 483, 92-95.

Fu, M., and Zuo, Y. (2011). Experiencedependent structural plasticity in the cortex. Trends Neurosci. 34, 177-187.

Gainey, M. A., Hurvitz-Wolff, J. R., Lambo, M. E., and Turrigiano, G. G. (2009). Synaptic scaling requires the GluR2 subunit of the AMPA receptor. J. Neurosci. 29, 6479-6489.

Galli, L., and Maffei, L. (1988). Spontaneous impulse activity of rat retinal ganglion cells in prenatal life. Science 242, 90-91.

Garaschuk, O., Hanse, E., and Konnerth, A. (1998). Developmental profile and synaptic origin of early network oscillations in the CA1 region of rat neonatal hippocampus. J. Physiol. 507, 219-236.

Geschwind, D. H., and Levitt, P. (2007). Autism spectrum disorders: developmental disconnection syndromes. Curr. Opin. Neurobiol. 17, 103-111.

Goodman, C. S., and Shatz, C. J. (1993). Developmental mechanisms that generate precise patterns of neuronal connectivity. Cell 72, 77-98.

Govindarajan, A., Israely, I., Huang, S. Y., and Tonegawa, S. (2011). The dendritic branch is the preferred integrative unit for protein synthesis-dependent LTP. Neuron $69,132-146$

Govindarajan, A., Kelleher, R. J., and Tonegawa, S. (2006). A clustered plasticity model of long-term memory engrams. Nat. Rev. Neurosci. 7, 575-583.

Hanse, E., Taira, T., Lauri, S., and Groc, L. (2009). Glutamate synapse in developing brain: an integrative perspective beyond the silent state. Trends Neurosci. 32, 532-537.

Harvey, C. D., and Svoboda, K. (2007). Locally dynamic synaptic learning rules in pyramidal neuron dendrites. Nature 450, 1195-1200.

Harvey, C. D., Yasuda, R., Zhong, H., and Svoboda, K. (2008). The spread of Ras activity triggered by activation of a single dendritic spine. Science 321, 136-140.

Hausser, M., and Mel, B. (2003). Dendrites: bug or feature? Curr. Opin. Neurobiol. 13, 372-383.
Hausser, M., Spruston, N., and Stuart, G. J. (2000). Diversity and dynamics of dendritic signaling. Science 290, 739-744.

Hofer, S. B., Mrsic-Flogel, T. D., Bonhoeffer, T., and Hubener, M. (2006). Lifelong learning: ocular dominance plasticity in mouse visual cortex. Curr. Opin. Neurobiol. $16,451-459$.

Hua, J. Y., and Smith, S. J. (2004). Neural activity and the dynamics of central nervous system development. Nat. Neurosci. 7, 327-332.

Huberman, A. D., Feller, M. B., and Chapman, B. (2008). Mechanisms underlying development of visual maps and receptive fields. Annu. Rev. Neurosci. 31, 479-509.

Jia, H., Rochefort, N. L., Chen, X., and Konnerth, A. (2010). Dendritic organization of sensory input to cortical neurons in vivo. Nature 464, 1307-1312.

Karmarkar, U. R., and Dan, Y. (2006). Experience-dependent plasticity in adult visual cortex. Neuron 52, 577-585.

Katz, L. C., and Shatz, C. J. (1996). Synaptic activity and the construction of cortical circuits. Science 274, 1133-1138.

Kelleher, R. J. III, Govindarajan, A., Jung, H. Y., Kang, H., and Tonegawa, S. (2004a). Translational control by MAPK signaling in long-term synaptic plasticity and memory. Cell 116, 467-479.

Kelleher, R. J. III, Govindarajan, A., and Tonegawa, S. (2004b). Translational regulatory mechanisms in persistent forms of synaptic plasticity. Neuron $44,59-73$.

Kleindienst, T., Winnubst, J., RothAlpermann, C., Bonhoeffer, T., and Lohmann, C. (2011). Activitydependent clustering of functional synaptic inputs on developing hippocampal dendrites. Neuron 72, 1012-1024.

Kole, M. H., and Stuart, G. J. (2012). Signal processing in the axon initial segment. Neuron 73, 235-247.

Larkum, M. E., and Nevian, T. (2008). Synaptic clustering by dendritic signalling mechanisms. Curr. Opin. Neurobiol. 18, 321-331.

Liao, D., Hessler, N. A., and Malinow, R. (1995). Activation of postsynaptically silent synapses during pairing-induced LTP in CA1 region of hippocampal slice. Nature 375, 400-404.

Losonczy, A., and Magee, J. C. (2006). Integrative properties of radial oblique dendrites in hippocampal
CA1 pyramidal neurons. Neuron 50 , 291-307.

Losonczy, A., Makara, J. K., and Magee, J. C. (2008). Compartmentalized dendritic plasticity and input feature storage in neurons. Nature 452 , 436-441.

Makino, H., and Malinow, R. (2011) Compartmentalized versus global synaptic plasticity on dendrites controlled by experience. Neuron 72 , 1001-1011.

McBride, T. J., Rodriguez-Contreras, A., Trinh, A., Bailey, R., and DeBello, W. M. (2008). Learning drives differential clustering of axodendritic contacts in the barn owl auditory system. J. Neurosci. 28, 6960-6973.

Meister, M., Wong, R. O. L., Baylor, D. A., and Shatz, C. J. (1991). Synchronous bursts of action potentials in ganglion cells of the developing mammalian retina. Science 252, 939-943.

Mrsic-Flogel, T. D., Hofer, S. B., Creutzfeldt, C., Cloez-Tayarani, I., Changeux, J. P., Bonhoeffer, T., and Hubener, M. (2005). Altered map of visual space in the superior colliculus of mice lacking early retinal waves. J. Neurosci. 25 , 6921-6928.

Murakoshi, H., Wang, H., and Yasuda, R. (2011). Local, persistent activation of Rho GTPases during plasticity of single dendritic spines. Nature $472,100-104$

Nevian, T., Larkum, M. E., Polsky, A., and Schiller, J. (2007). Properties of basal dendrites of layer 5 pyramidal neurons: a direct patch-clamp recording study. Nat. Neurosci. 10, 206-214.

O'Donovan, M., Ho, S., and Yee, W. (1994). Calcium imaging of rhythmic network activity in the developing spinal cord of the chick embryo. J. Neurosci. 14, 6354-6369.

Poirazi, P., Brannon, T., and Mel, B. W. (2003). Pyramidal neuron as twolayer neural network. Neuron 37, 989-999.

Poirazi, P., and Mel, B. W. (2001). Impact of active dendrites and structural plasticity on the memory capacity of neural tissue. Neuron 29 , 779-796.

Polsky, A., Mel, B. W., and Schiller, J. (2004). Computational subunits in thin dendrites of pyramidal cells. Nat. Neurosci. 7, 621-627.

Sanes, J. R., and Yamagata, M. (2009). Many paths to synaptic specificity. Annu. Rev. Cell Dev. Biol. 25, 161-195.

Schiller, J., Schiller, Y., Stuart, G., and Sakmann, B. (1997). Calcium action potentials restricted to distal apical dendrites of rat neocortical pyramidal neurons. J. Physiol. 505(Pt 3), 605-616.

Steward, O., and Levy, W. B. (1982). Preferential localization of polyribosomes under the base of dendritic spines in granule cells of the dentate gyrus. J. Neurosci. 2 , 284-291.

Steward, O., and Schuman, E. M. (2003). Compartmentalized synthesis and degradation of proteins in neurons. Neuron 40, 347-359.

Takahashi, N., Kitamura, K., Matsuo, N., Mayford, M., Kano, M., Matsuki, N., and Ikegaya, Y. (2012). Locally synchronized synaptic inputs. Science 335, 353-356.

Takahashi, T., Svoboda, K., and Malinow, R. (2003). Experience strengthening transmission by driving AMPA receptors into synapses. Science 299, 1585-1588.

Torborg, C. L., Hansen, K. A., and Feller, M. B. (2005). High frequency, synchronized bursting drives eyespecific segregation of retinogeniculate projections. Nat. Neurosci. 8 , 72-78.

Varga, Z., Jia, H., Sakmann, B., and Konnerth, A. (2011). Dendritic coding of multiple sensory inputs in single cortical neurons in vivo. Proc. Natl. Acad. Sci. U.S.A. 108, 15420-15425

Weliky, M., and Katz, L. C. (1997). Disruption of orientation tuning in visual cortex by artificially correlated neuronal activity. Nature 386, 680-685.

Wong, R. O. L. (1999). Retinal waves and visual system development. Annu. Rev. Neurosci. 22, 29-47.

Wu, G. Y., Deisseroth, K., and Tsien, R. W. (2001). Spaced stimuli stabilize MAPK pathway activation and its effects on dendritic morphology. Nat. Neurosci. 4, 151-158.

Wu, X. E., and Mel, B. W. (2009). Capacity-enhancing synaptic learning rules in a medial temporal lobe online learning model. Neuron 62 , 31-41.

Xu, H. P., Furman, M., Mineur, Y. S., Chen, H., King, S. L., Zenisek, D. Zhou, Z. J., Butts, D. A., Tian, N., Picciotto, M. R., and Crair, M. C. (2011). An instructive role for patterned spontaneous retinal activity in mouse visual map development. Neuron 70, 1115-1127.

Yuste, R. (2011). Dendritic spines and distributed circuits. Neuron 71 , 772-781.

Yuste, R., Peinado, A., and Katz, L. C. (1992). Neuronal domains in 
developing neocortex. Science. 257, 666-669.

Zhu, J. J., Qin, Y., Zhao, M., Van, A. L., and Malinow, R. (2002). Ras and Rap control AMPA receptor trafficking during synaptic plasticity. Cell 110, 443-455.

Zoghbi, H. Y. (2003). Postnatal neurodevelopmental disorders: meeting at the synapse? Science 302, 826-830.

Conflict of Interest Statement: The authors declare that the research was conducted in the absence of any commercial or financial relationships that could be construed as a potential conflict of interest.
Received: 15 March 2012; paper pending published: 30 March 2012; accepted: 14 May 2012; published online: 31 May 2012.

Citation: Winnubst J and Lohmann C (2012) Synaptic clustering during development and learning: the why, when, and how. Front. Mol. Neurosci. 5:70. doi: 10.3389/fnmol.2012.00070
Copyright (C) 2012 Winnubst and Lohmann. This is an open-access article distributed under the terms of the Creative Commons Attribution Non Commercial License, which permits non-commercial use, distribution, and reproduction in other forums, provided the original authors and source are credited. 